\title{
Global Evolution of Obesity Research in Children and Youths: Setting Priorities for Interventions and Policies
}

\author{
Bach Xuan Tran ${ }^{a}$ b Kim Anh Dang ${ }^{a}$ Huong Thi Le ${ }^{a}$ Giang Hai Hac \\ Long Hoang Nguyen ${ }^{d}$ Tu Huu Nguyen ${ }^{e}$ Tung Hoang Tran ${ }^{f}$ \\ Carl A. Latkin ${ }^{b}$ Cyrus S.H. Ho ${ }^{g}$ Roger C.M. Ho ${ }^{d, h}$ \\ a Institute for Preventive Medicine and Public Health, Hanoi Medical University, Hanoi, \\ Vietnam; ${ }^{b}$ Bloomberg School of Public Health, Johns Hopkins University, Baltimore, MD, \\ USA; ' Institute for Global Health Innovations, Duy Tân University, Da Nang, Vietnam; \\ ${ }^{d}$ Center of Excellence in Behavioral Medicine, Nguyen Tat Thanh University, Ho Chi Minh

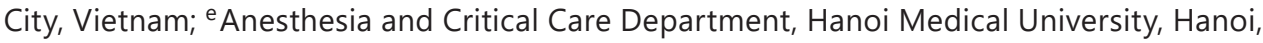

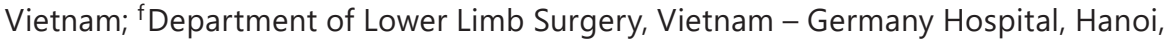 \\ Vietnam; ${ }^{9}$ Department of Psychological Medicine, National University Hospital, Singapore, \\ Singapore; hepartment of Psychological Medicine, Yong Loo Lin School of Medicine, \\ National University of Singapore, Singapore, Singapore
}

\section{Keywords}

Scientometrics · Child obesity · Global evolution · Mapping

\begin{abstract}
Background: Childhood obesity has become a major global epidemic that causes substantial social and health burdens worldwide. The effectiveness of childhood obesity control and prevention depends largely on understanding the issue, including its current development and associated factors in a contextualized perspective. Objectives: Our study aimed to gauge this kind of understanding. Methods: We systematically searched the Web of Science database for studies concerning child obesity published up to 2017 and analyzed the volume of publications, growth rates, impact scores, collaborations, authors, affiliations, and journals. A total of 57,444 research papers were included. Results: The three subject categories with the highest number of papers (over 3,000) were (1) nutrition and dietetics, (2) pediatrics, and (3) public, environmental, and occupational health. We found a dramatic increase in the amount of scientific literature on childhood obesity in the past one or two decades, led by scholars from the USA - ranking at the top regarding the total number of papers $(23,965$ papers; $30.8 \%)$ and total number of citations (859,793 citations) - and multiple Western countries where the obesity epidemic is prevalent. Conclusions: The findings highlight the need for improving international and local research capacities and collaboration to accelerate knowledge production and translation into contextualized and effective childhood obesity prevention.
\end{abstract}


Tran et al.: Global Evolution of Obesity Research in Children and Youths

\section{Introduction}

Childhood obesity has become a major global epidemic that imposes a substantial social and health burden worldwide [1,2]. An estimated 4.0 million deaths and 120 million disabilityadjusted life years (DALYs) in 2015 were attributable to an excess body mass index (BMI) globally [3]. Obesity in childhood is a special concern, given its life course impact by causing the development of multiple chronic conditions. Specifically, children who are overweight are more likely to become obese at a later age $[4,5]$, have a deteriorated quality of life and significantly higher health care costs, and live less long [6, 7]. Previous studies have documented associations of childhood obesity with the development of musculoskeletal disorders, hypertension, cardiovascular disease, and diabetes $[5,8]$. Furthermore, overweight children have poorer mental health as a result of having low self-esteem, body image disturbance, and depression $[5,9]$.

The childhood obesity epidemic has been expanding in both developed and developing countries. According to the World Health Organization (WHO), 381 million children under 20 years were overweight or obese in 2016 [2, 5, 10]. Over the past four decades, the prevalence of excess weight in both male and female adolescents has been substantially increasing (from 4 to over 18\%) [10]. Curbing this continuing epidemic requires strong global political commitment, involvement of stakeholders, and synergetic efforts from nations and communities in effectively delivering preventive and management strategies. The United Nations have acted to advance the implementation of global commitment by launching the WHO Global Strategy on Diet, Physical Activity and Health [11, 12]. In 2016, a report by the Commission on Ending Childhood Obesity suggested six recommendations for obesity control at global, regional and local levels; it was presented at the World Health Assembly in 2017 [13].

Implementing these recommendations requires enormous amounts of evidence, but the availability and applicability of evidence are heterogeneous across settings. Many developing countries, where there is a double burden of malnutrition, are not sufficiently capable of producing timely evidence to inform policy development $[14,15]$. In addition, the translation of international research findings into a specific setting may be confined by many sociocultural and structural barriers and may face challenges in maintaining a long-term impact of interventions $[2,10,16]$. Thus, exploring any evidence gaps and institutions with sufficient networks and capacities is vital to ensure the effectiveness and substantiality of further interventions in the field of childhood obesity.

One method that would be appropriate for measuring these gaps is scientometric analysis [17]. This method objectively measures the international and national interest in specific research topics through examining tendencies in published articles over time and networks of research collaborations in literature databases $[18,19]$. By offering such insights, this approach fosters effective government investments in research [20]. To date, several scientometric studies on obesity have been conducted, but they only focused on people in the Middle East or India [21, 22]. None of them provided evidence about global research on childhood obesity. Therefore, we aimed to analyze the global growth of research production and patterns of research areas in the field of childhood obesity.

\section{Methods}

\section{Bibliographic Database}

The Web of Science database (WoS) was used for searching, which is the oldest database covering citation and bibliographic data since 1900 [23]. WoS provides more comprehensive 
data (such as author information, journal, and publication year) that allow for in-depth analysis than other databases like Scopus and MEDLINE. Articles in WoS are tagged according to document type, such as review articles or original articles, following a standardized structure, which is vital for a literature analysis [24].

In this study, we also collected DALYs (rate) and BMI data from the Global Burden of Diseases website (http://ghdx.healthdata.org/gbd-results-tool). We extracted only data from 2016 regardless of sex, age group, and location, due to inconsistencies in year ranges.

\section{Search Strategy}

The WHO's definition of childhood obesity and other previous studies were adopted to build the search strategy [5, 9, 25-27], focusing on (1) child and (2) obesity. The search strategy was as follows and as displayed in online supplementary Table S1 (for all online suppl. material, see www.karger.com/doi/10.1159/000497121):

1. Child* OR Pediatric* OR Paediatric* OR Infant* OR newborn* OR bab* OR Toddler* OR pre-adolescen* OR preadolescen* OR Adolescen* OR Youth* OR Youngster* OR Teen* OR Teenager* OR teenage OR preschooler* OR pre-schooler* OR pre-school* OR preschool* OR "Pre-school child*" OR "school-aged child*" OR schoolchild* OR "school age*” OR Schoolage* OR "nursery school*" OR kindergar* OR "primary school*" OR "secondary school*" OR "grade school*" OR "elementary school*” OR "high school*" OR highschool*

2. Overweight OR "Over weight" OR Preobes* OR Pre-obes* OR Obes* OR overnutrition

OR "excessive fat accumulation" OR "fat levels" OR "excess body fat" OR "weight

disorder" OR "High body mass index"

Only research articles and review articles were included in our study, while others were excluded. Papers published from January 1, 2018, onward were excluded due to the incomplete bibliometric data for that year. Documents having anonymous authors and papers written in languages other than English were also removed.

\section{Data Extraction}

The exported data included: (1) title and abstract; (2) authors' names and their affiliations; (3) citations for each paper; (4) institutional affiliations; (5) year of publication; (6) author keywords; and (7) research area.

\section{Data Analysis}

The data included information on general characteristics (total publications, publication year, number of authors, and main category); the most prolific authors (more than 100 papers); the countries and institutions/organizations with the highest total number of publications; journal details and highly impactful papers (times cited: more than 1,300 times); and keywords (most common keywords and co-occurrence of keywords). Then, we synthesized the data on the number of publications and DALYs in order to show whether the research productivity in each country aligned with the disease burden regarding obesity [3].

As there were differences in countries' names between the databases (e.g., "USA" in WoS and "United States" in the Global Burden of Disease Study data), one of the authors (G.H.H.) checked for inconsistencies, while another one (L.H.N.) verified the data. Any discrepancies between the two researchers were resolved via discussion.

Overviews of the collaborations from the most productive countries as well as the most cited papers were created using the VOSviewer software (http://www.vosviewer.com/). A visualization map represented collaborations and contributions from 110 countries using a threshold of 10 or more publications. We also used this software to generate co-occurrence networks of keywords by using keyword frequency (218 keywords with a minimum of 100 
Fig. 1. Selection of papers.

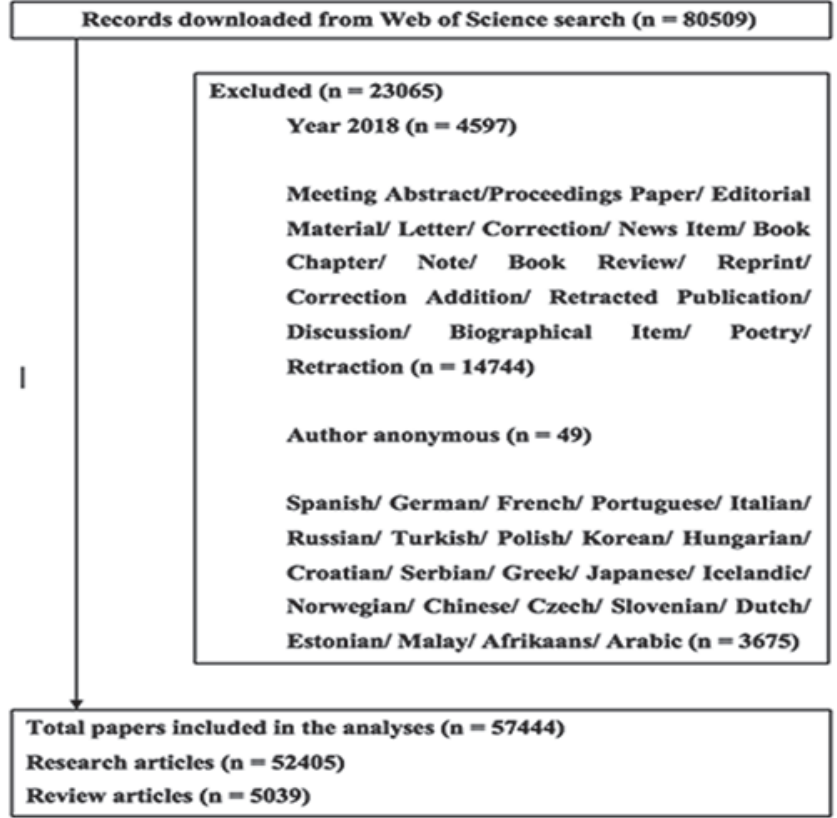

appearances). The thresholds were chosen based on our experiments with VOSviewer to achieve a presentation most appropriately reflecting a global overview of collaborations. The 2016 population data were collected from the World Bank website (https://data.worldbank. org/indicator/SP.POP.TOTL) to calculate the number of publications per million inhabitants. For Wales, Scotland, and Northern Ireland, we used the Office for National Statistics Great Britain to obtain the population data (https://www.ons.gov.uk/peoplepopulationandcommunity/populationandmigration/populationestimates).

\section{Results}

\section{Numbers of Papers and Publication Trends}

The study selection process is shown in Figure 1. There were 80,509 studies published between 1920 and 2017. After removing 23,065 studies that did not match the eligibility criteria, a total of 57,444 research papers were included in the analysis, consisting of 52,405 research articles and 5,039 review articles.

Figure 2 illustrates the general trend of the search results. Since 1970, the volume of research articles has increased dramatically every 10 years. Noticeably, approximately $63 \%$ of all publications were published in the period from 2011 to 2017. Regarding the location of the first author, the greatest number of publications in childhood obesity research came from the USA ( $n=21,045 ; 36.6 \%)$, followed by England $(n=3,168 ; 5.5 \%)$. Papers with $4-6$ authors accounted for the largest proportion (41.9\%) (Fig. 3; online suppl. Table S1).

\section{Journal Diversity and Author Collaborations}

The majority of articles were classified as having $1(64.3 \%)$ or $2(28.7 \%)$ journal subject categories (online suppl. Table S1). The most common subject categories are displayed in Figure 4. There were 6 subject categories with regard to childhood obesity being covered by more than 3,000 papers per category, including: nutrition and dietetics; pediatrics; public, 
Tran et al.: Global Evolution of Obesity Research in Children and Youths

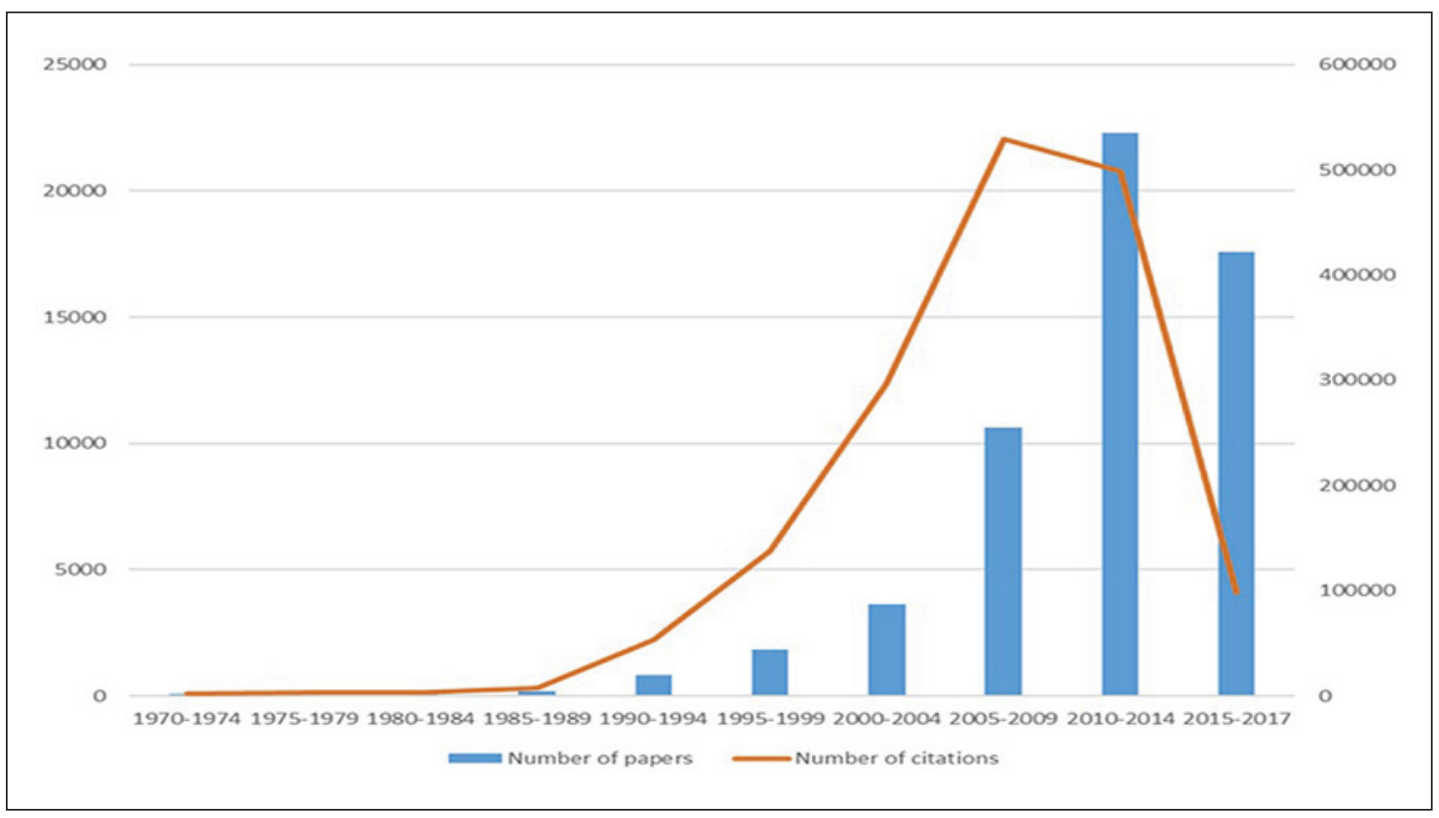

Fig. 2. Numbers of papers and citations in different time periods.

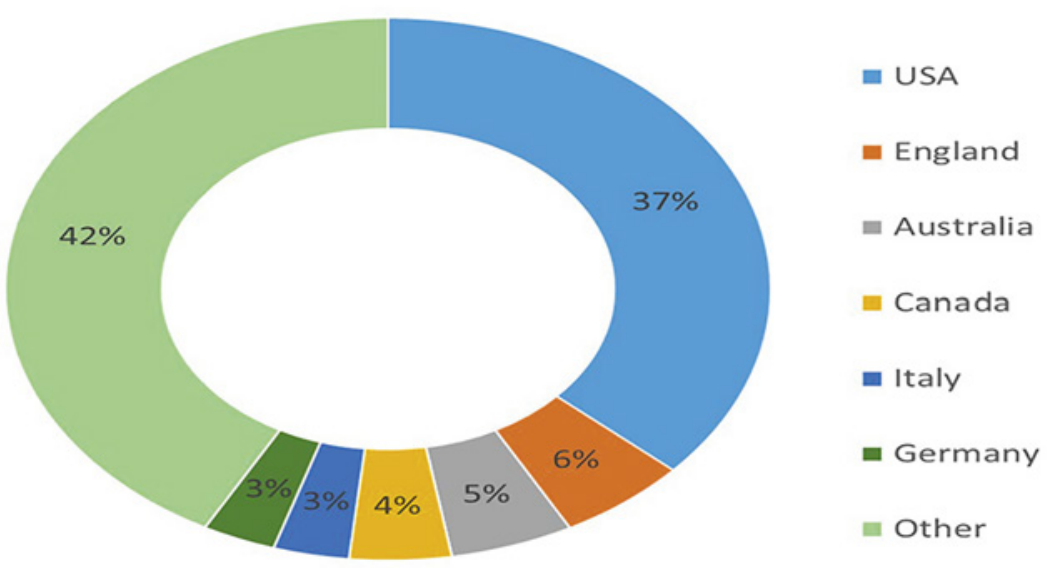

Fig. 3. Countries of origin of the first authors.

environmental, and occupational health; endocrinology and metabolism; general and internal medicine; and psychology. The International Journal of Obesity had the highest number of papers in the field of nutrition and dietetics $(n=1,445)$ and endocrinology and metabolism ( $n=1,445$ papers) (online suppl. Table S2).

Table 1 shows that the collaboration index (the average number of authors per article) was generally increasing during the period, with the highest index (6.42) seen in 2017. Most of the first authors were from North American countries (USA and Canada) and Europe (England, Spain, Greece, Germany, Belgium, Hungary, Denmark, The Netherlands, and Italy). 
Tran et al.: Global Evolution of Obesity Research in Children and Youths

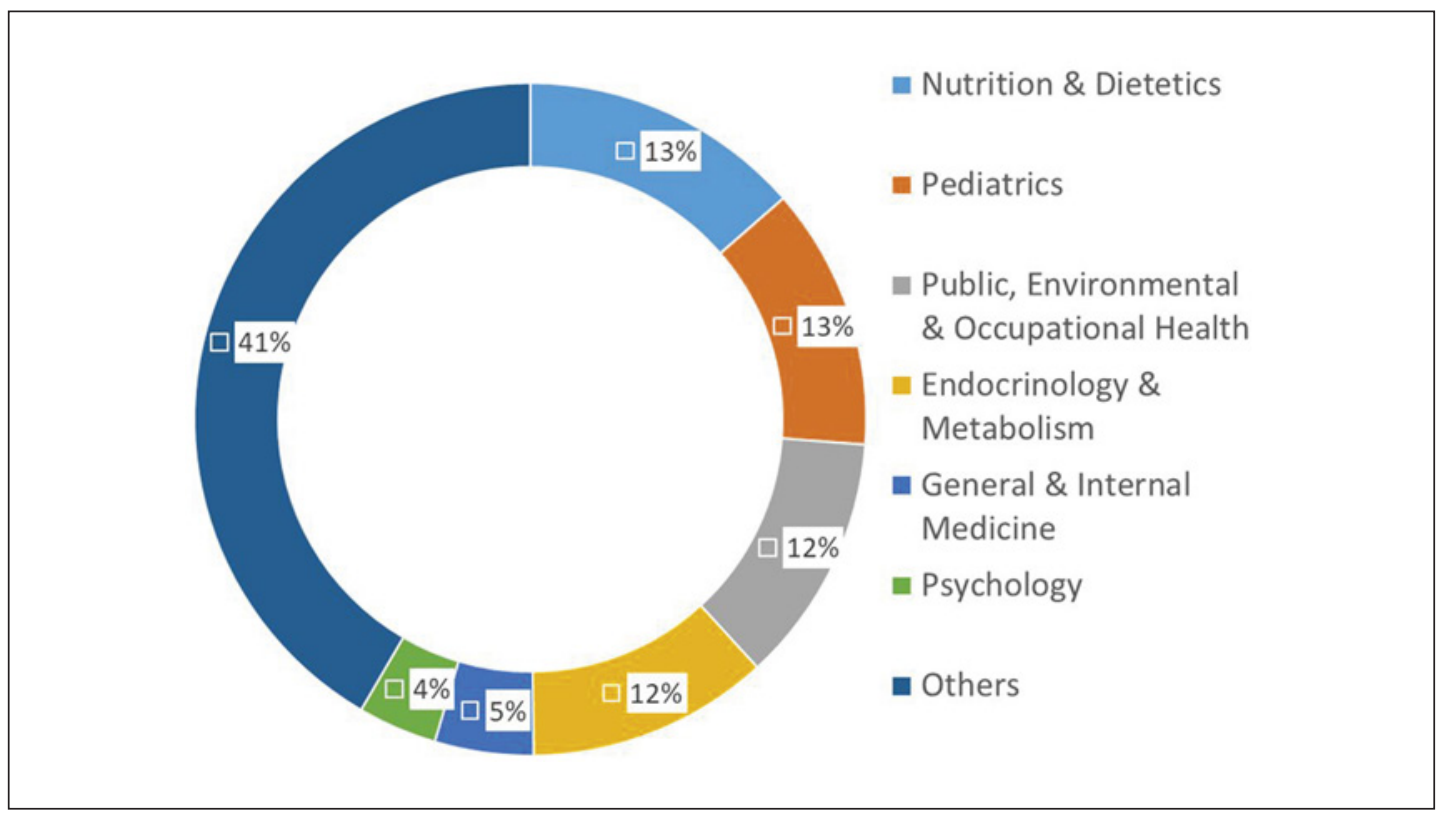

Fig. 4. The most common research areas.

Table 1. Collaboration index of researchers in the field of child obesity

\begin{tabular}{ll}
\hline Year & Collaboration index \\
\hline 2000 & 4.92 \\
2001 & 4.53 \\
2002 & 4.57 \\
2003 & 4.94 \\
2004 & 4.80 \\
2005 & 4.79 \\
2006 & 4.89 \\
2007 & 5.01 \\
2008 & 5.02 \\
2009 & 5.28 \\
2010 & 5.11 \\
2011 & 5.25 \\
2012 & 5.40 \\
2013 & 5.56 \\
2014 & 5.69 \\
2015 & 5.88 \\
2016 & 6.25 \\
2017 & 6.42 \\
\hline
\end{tabular}

The country with the highest number of most productive authors was the USA with 11 authors (online suppl. Table S3). The total volume of papers published by the most productive authors varied from 108 to 285, and the total number of citations ranged from 3,701 to 16,076. L.A. Moreno from Spain was the author who had published the highest total number of papers $(n=285)$ and the highest number of papers in collaboration $(n=284)$. However, S.R. Daniels from the University of Cincinnati College of Medicine, Cincinnati, OH, USA, led regarding the total number of citations $(n=16,076)$, while G.S. Berenson from the Tulane School of Public Health and Tropical Medicine, New Orleans, LA, USA, had the highest number of citations per paper $(n=118.57$ ) (online suppl. Table S3). 


\section{Country Collaborations}

Table 2 shows that the 5 countries ranking at the top in productivity were from North America (the USA with 23,965 papers and Canada with 3,441 papers), East Asia and Pacific (Australia with 3,939 papers), and Europe (England with 5,150 papers and Germany with 2,510 papers); all of them are high-income countries. The USA led and ranked first in the total number of papers $(n=23,965 ; 30.8 \%)$, total number of citations $(n=859,793)$, and distinct countries of collaboration $(n=175)$. The main collaborations of authors from the USA were with authors from England ( $n=868$ papers). England ranked second in the total number of papers $(5,150$ papers $)$ and was the primary partner of the USA, Australia, and European countries (Denmark, Finland, and Scotland). Among Asian countries, the People's Republic of China had the highest research productivity (a total of 2,273 papers), followed by Japan (a total of 1,076 papers) and South Korea (a total of 883 papers). The number of papers per million inhabitants was lowest in India with 0.9 papers per million inhabitants, whereas Denmark ranked first with 182.5 papers per million inhabitants (Table 2).

Figure 5 displays a visual map of the research contributions of the top 110 countries with a minimum of 10 papers. The USA accounted for the largest proportion of contributors and mainly collaborated with England, Canada, Australia, and the People's Republic of China. Several countries in Western Europe, such as Germany, Spain, Belgium, Italy, and France, were in the same cluster of collaboration. Clusters were also seen among the Netherlands, Norway, and Switzerland, as well as East and South Asian countries such as Singapore, Thailand, Taiwan, the People's Republic of China, Japan, and India (Fig. 5).

The USA had the largest total number of papers, and the DALYs (rate) attributable to a high BMI (online suppl. Table S4) were also high in general as well as for both males and females (44.2 and 55.3, respectively). Although Brazil ranked 6th, Turkey ranked 14th, and Iran ranked 15th regarding the total number of papers, the DALYs in these countries were significantly higher than in other countries among the top 20. South Korea had a high percentage of overweight/obese children in 2016 compared to other top 20 countries, but its DALYs were low. Several Western European countries such as Germany, Spain, Belgium, Italy, and France, which were in the same cluster of collaboration, had a high prevalence of overweight/obesity among both genders in 2016 (online suppl. Table S4).

\section{Keyword Analysis}

Figure 6 illustrates the co-occurrence of papers' keywords. Overall, there were 39,397 keywords, of which 218 keywords were used 100 times or more. We assigned those to four groups:

1. Obesity classification: information about BMI, adiposity, weight gain, and birth weight

2. Consequences of childhood obesity: metabolic syndrome, hypertension, insulin resistance, and blood pressure

3. Effects: depression, body image, and weight loss as the effects of obesity on adolescents

4. Interventions related to childhood obesity: health promotion, physical activity, diet, and parent support

The relationship between mental health (depression or stress) and childhood obesity was not receiving suitable attention. Depression and stress appeared 361 times $(0.92 \%)$ and 136 times $(0.34 \%)$, respectively. Although obesity is not a mental health disorder, its impact on the psychological health of those living with obesity, especially children or adolescents, should be considered [28]. Meanwhile, the "intervention" keywords showed that the most common topics of interventions were physical activity, education, food intake, and diet with the support of parents, and that the main subjects were students and youths. 
Tran et al.: Global Evolution of Obesity Research in Children and Youths

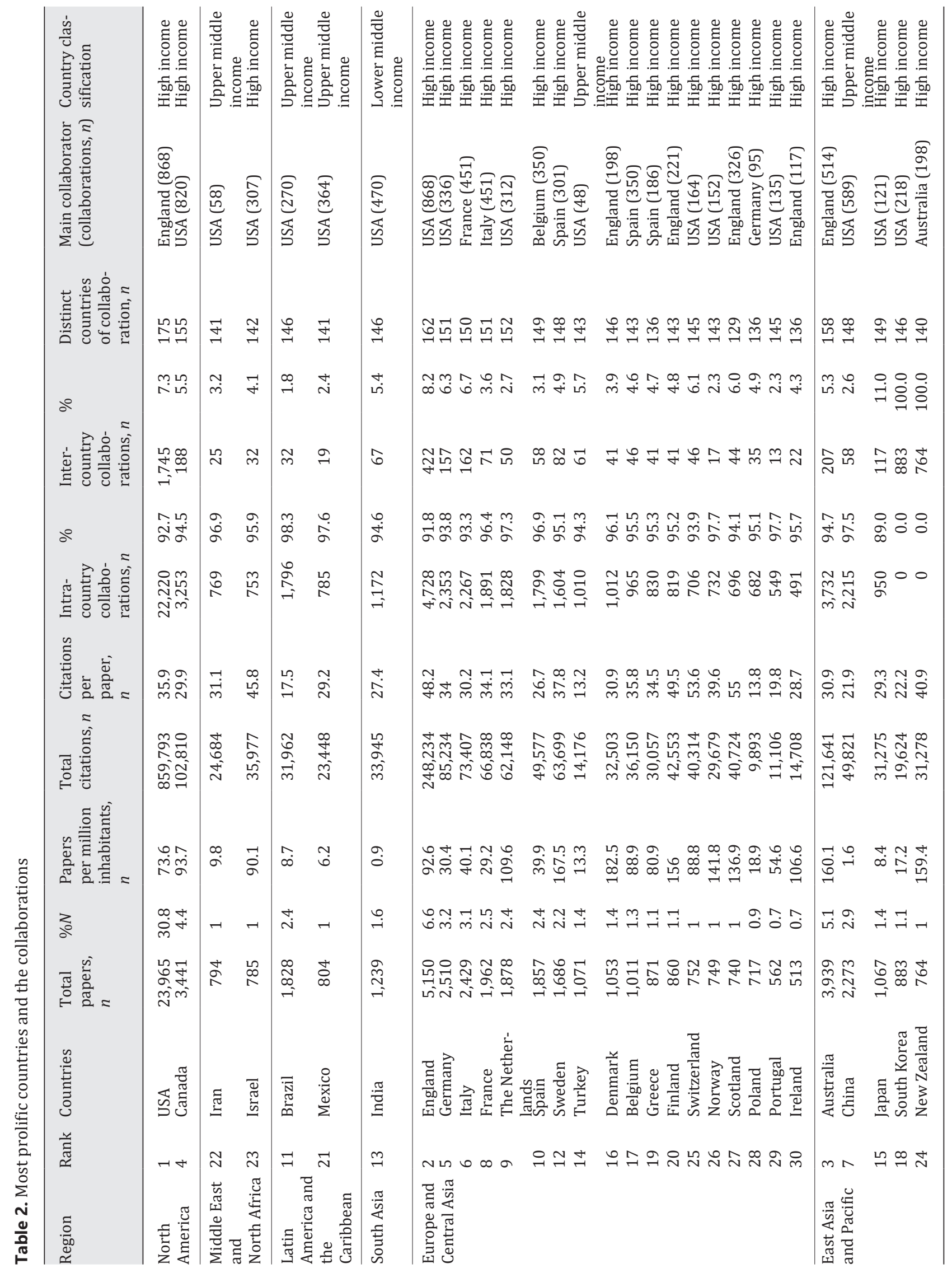




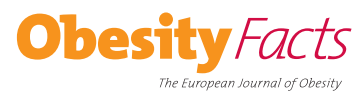

\begin{tabular}{l|l}
\hline Obes Facts 2019;12:137-149 \\
\hline DOI: 10.1159/000497121 & $\begin{array}{l}\text { @ 2019 The Author(s). Published by S. Karger AG, Basel } \\
\text { www.karger.com/ofa }\end{array}$ \\
\hline
\end{tabular}

Tran et al.: Global Evolution of Obesity Research in Children and Youths

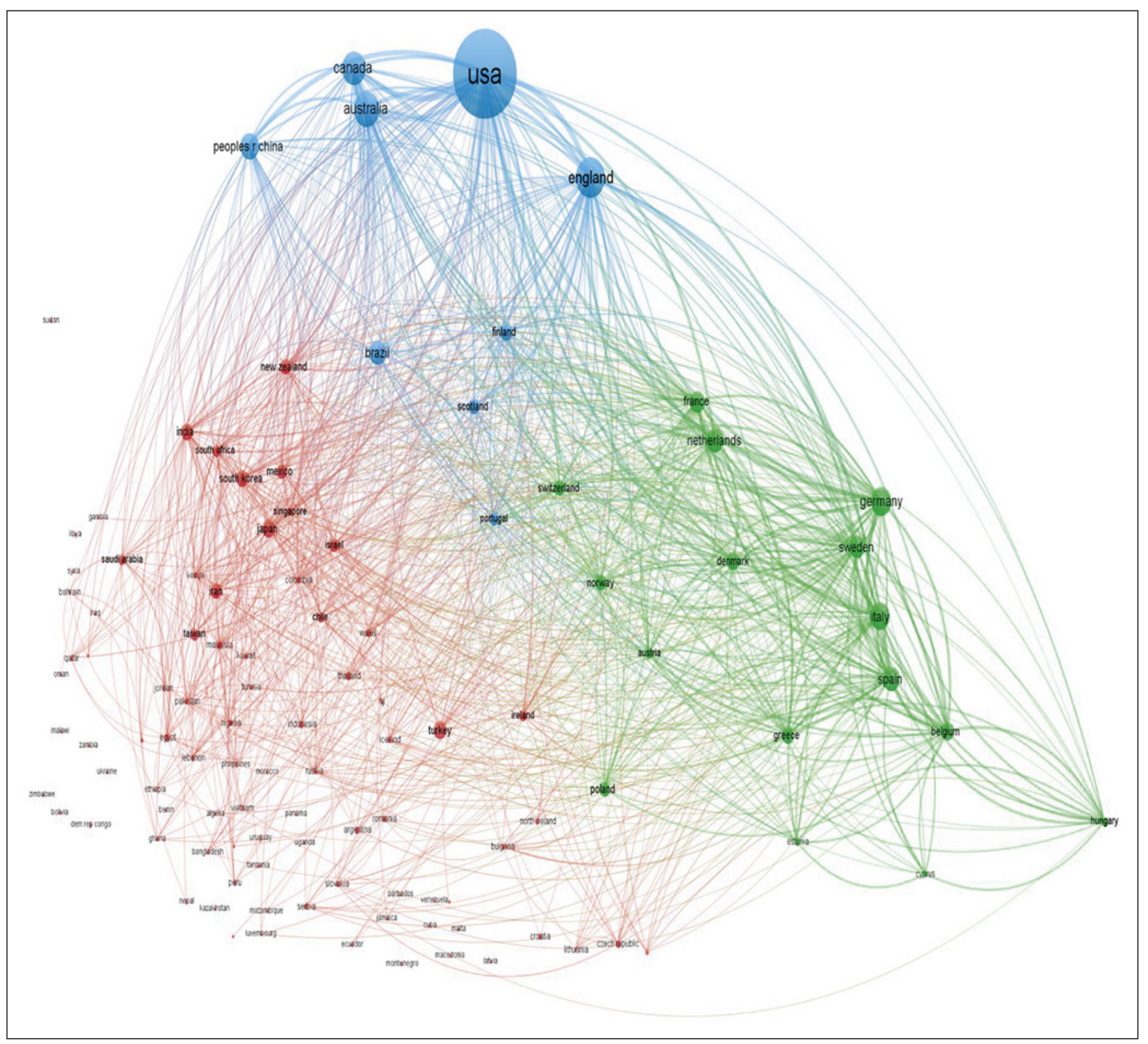

Fig. 5. Global networks of the most prolific countries.

\section{Discussion}

This review summarized the global research development in the field of childhood obesity. We found a dramatic increase in the amount of scientific literature on childhood obesity in the past two decades, led by scholars from the USA and Western countries, where the obesity epidemic is prevalent. The clusters of collaboration between countries in childhood obesity research were mainly based on cultural similarity and geographic proximity. There was a disproportionality between the number of publications and the burden of childhood obesity in several countries, showing potential knowledge gaps regarding interventions. Furthermore, the major part of the research focused on assessing excess weight status and 
Tran et al.: Global Evolution of Obesity Research in Children and Youths

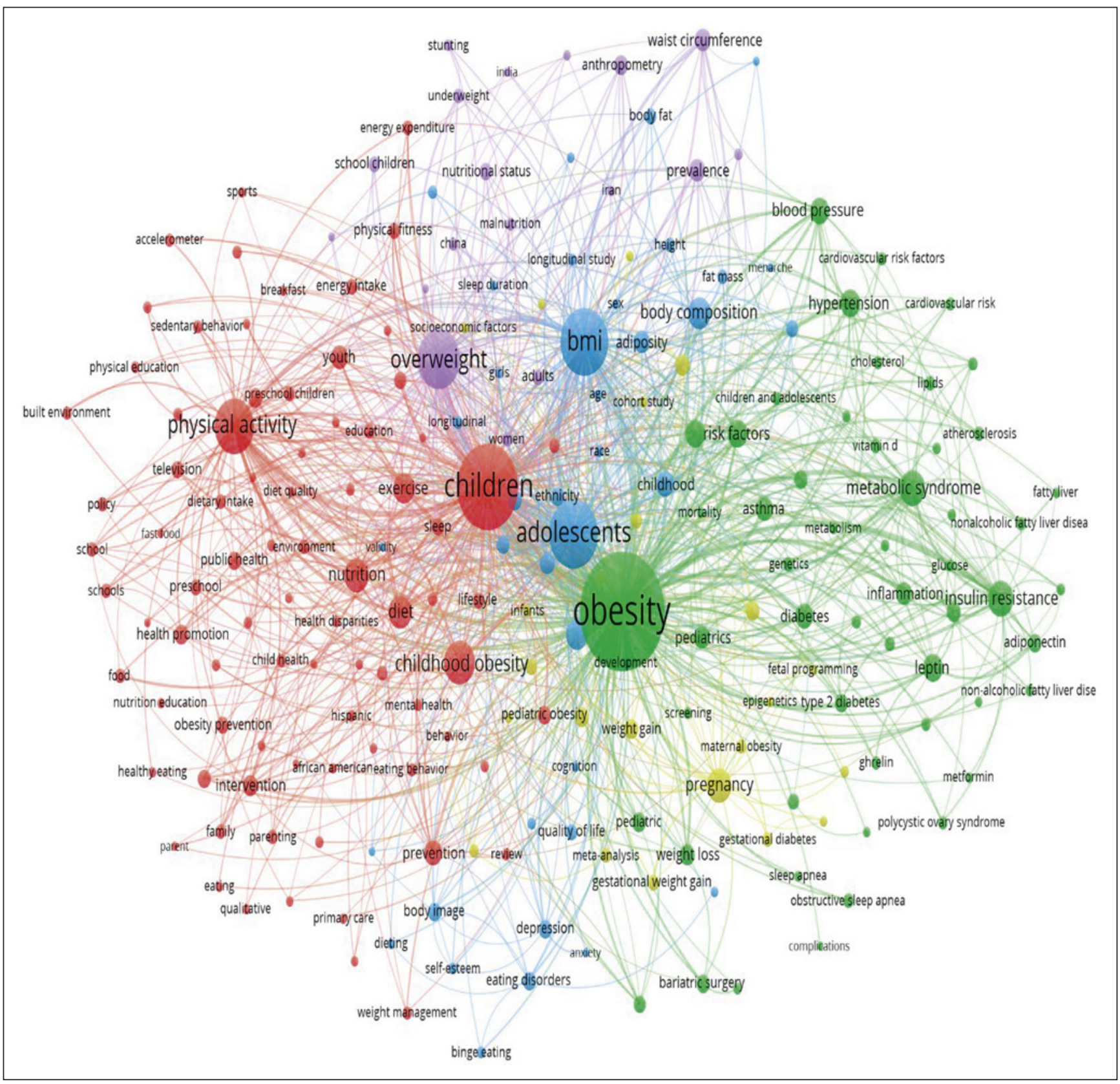

Fig. 6. Co-occurrence of authors' keywords.

its relation to the physical and mental health of children and adolescents, while research on interventions, especially large-scale, contextualized measures, is still limited.

Our results highlighted that the USA were the global knowledge hub in the field of childhood obesity, and the strongest collaborations worldwide were between the USA, Australia, England, and Canada, which is similar to the results of previous studies in different research areas $[29,30]$. This may be explained by the fact that there was a positive relationship between a high prevalence of obesity and a high socioeconomic status of the population among high-income countries compared to low- and middle-income countries $[1,2]$. 
Moreover, the period from 2000 until now witnessed a significant growth of the number of publications in the field of childhood obesity. The prevalence of overweight and obesity also increased dramatically in this period due to the rise in food consumption, nutritional transition [31], and marketing by the food industry [32]; for instance, the overconsumption of fast-food and sugary products, increasing total daily calorie intake, is notoriously correlated to a rising BMI [33]. In addition to diet, urbanization was a factor contributing to the growth of childhood obesity, including a sedentary lifestyle, reduced physical activities, and the rapid proliferation of the Internet [34, 35].

In 2015, at the global level, a high BMI ( $>30$ ) contributed to approximately $36 \%$ of DALYs and excess weight was related to 120 million (84-158 million) DALYs among adults [3]. However, in several countries, although the DALYs related to excess weight were high, the research output was not highly correlated. Although the WHO reported that in 2016 about half of the children with excess weight lived in Asia, the partnerships of cluster cooperations between Northern America, Western Europe, and East and South Asia might not be sufficient. This may create gaps in the global network literature due to the demands of understanding contextual factors and multilevel sociobiological determinants. Therefore, it is necessary to improve local research capacities and international research collaborations in countries hit hard by the obesity epidemic. Although science is on the rise in developing countries, their governments need to "fully support" the value of science to catch up with the developed world [36]. Also, multinational research networks involving different cultures, geographical areas, and socioeconomic subgroups should be strengthened.

This is the first bibliometric analysis of the global childhood obesity literature. Bibliometric and other, similar methods have been utilized for showing trends in different research fields using reliable, practical methods that measure, evaluate, and analyze scientific products $[18,19]$. In our data set, the approaches to childhood obesity in the current publications chiefly focused on prevalence, classification, risk factors for childhood obesity, and consequences related to metabolic syndrome. We also found that a small number of studies within the data set shifted their focus to the effect of obesity on psychology such as depression, anxiety, eating disorders and body image, and self-esteem. Depression and obesity among children are two primary public health issues. Several previous studies have revealed a significant association of obesity with depression and with depressive symptoms among adolescent $[37,38]$. Obesity may not directly result in depression but be related to experiences of shame and social isolation, which can lead to depression [38, 39]. However, because little attention has been paid to determining the prevalence of mental health issues among obese children, as well as to effective interventions, we suggest that more evidence regarding the effect of obesity on mental health should be gathered. In terms of prevention, most of the methods were based on increasing physical activity, promoting a healthy diet, and implementing educational programs. Furthermore, in our data set, when determining the 218 keywords with a minimum number of 100 appearances, only a small number of studies mentioned interventions based on the Internet. Previous research has highlighted the effectiveness of Internet-based obesity intervention programs among adolescents [40, 41]. Moreover, the relationships of obesity to mental health and wellness have not been fully investigated by the scientific community [42]. Obesity and mental health traditionally are treated separately; however, the relationship between them is bidirectional. Depression causes inactivity and thereby increases obesity. Obese individuals are frequently targeted for bullying at school, causing an increase in depressive symptoms [43].

Several limitations of our study should be considered. First, we only used the WoS database to collect scientific papers. Thus, there may be articles not provided by WoS which may influence the findings of our study. Second, because of the restriction of the scope of the search, we only included research articles and review articles; we excluded meeting abstracts, books, book 
Tran et al.: Global Evolution of Obesity Research in Children and Youths

chapters, and letters, which may have affected our results. We also excluded publications that were not written in English. Third, the analysis of occurrence and co-occurrence of keywords was based on the chosen keywords, and this can cause bias in terms of WoS-indexed papers.

In conclusion, this study presents the global research trends and developments in the field of childhood obesity. In addition, it highlights the needs for improving international and local research capacities and collaborations to accelerate knowledge production and translation into contextualized and effective childhood obesity prevention.

\section{Statement of Ethics}

The authors have no ethical conflicts to disclose.

\section{Disclosure Statement}

The authors have no conflicts of interest to declare.

\section{Author Contributions}

Bach Xuan Tran, Giang Hai Ha, Kim Anh Dang, Huong Thi Le, Carl A. Latkin, Long Hoang Nguyen, Cyrus S.H. Ho, and Roger C.M. Ho: conceptualization; Bach Xuan Tran, Giang Hai Ha, Kim Anh Dang, Huong Thi Le, Carl A. Latkin, Long Hoang Nguyen, Cyrus S.H. Ho, and Roger C.M. Ho: formal analysis; Bach Xuan Tran and Giang Hai Ha: investigation; Bach Xuan Tran, Giang Hai Ha, Kim Anh Dang, Huong Thi Le, Carl A. Latkin, Long Hoang Nguyen, Cyrus S.H. Ho, and Roger C.M. Ho: methodology; Bach Xuan Tran, Giang Hai Ha, Kim Anh Dang, Huong Thi Le, Carl A. Latkin, and Long Hoang Nguyen: project administration; Bach Xuan Tran, Giang Hai Ha, Kim Anh Dang, Huong Thi Le, Carl A. Latkin, Long Hoang Nguyen, Cyrus S.H. Ho, and Roger C.M. Ho: writing - original draft; Bach Xuan Tran, Giang Hai Ha, Kim Anh Dang, Huong Thi Le, Carl A. Latkin, Long Hoang Nguyen, Tu Huu Nguyen, Tung Hoang Tran, Cyrus S.H. Ho, and Roger C.M. Ho: writing - review and editing.

\section{References}

1 Bhurosy T, Jeewon R. Overweight and obesity epidemic in developing countries: a problem with diet, physical activity, or socioeconomic status? ScientificWorldJournal. 2014;2014:964236.

2 Caballero B. The global epidemic of obesity: an overview. Epidemiol Rev. 2007;29(1):1-5.

3 Afshin A, Forouzanfar MH, Reitsma MB, Sur P, Estep K, Lee A, et al.; GBD 2015 Obesity Collaborators. Health effects of overweight and obesity in 195 countries over 25 years. N Engl J Med. 2017 Jul;377(1):13-27.

4 Power C, Lake JK, Cole TJ. Measurement and long-term health risks of child and adolescent fatness. Int J Obes Relat Metab Disord. 1997 Jul;21(7):507-26.

5 World Health Organization. Childhood overweight and obesity [Internet]. Geneva: World Health Organization; 2016. Available from: https://www.who.int/dietphysicalactivity/childhood/en/.

6 Hoffmans MD, Kromhout D, de Lezenne Coulander C. The impact of body mass index of 78,612 18-year old Dutch men on 32-year mortality from all causes. J Clin Epidemiol. 1988;41(8):749-56.

7 Lifshitz F. Obesity in children. J Clin Res Pediatr Endocrinol. 2008;1(2):53-60.

8 Franks PW, Hanson RL, Knowler WC, Sievers ML, Bennett PH, Looker HC. Childhood obesity, other cardiovascular risk factors, and premature death. N Engl J Med. 2010 Feb;362(6):485-93.

9 Showell NN, Fawole O, Segal J, Wilson RF, Cheskin LJ, Bleich SN, et al. A systematic review of home-based childhood obesity prevention studies. Pediatrics. 2013 Jul;132(1):e193-200.

10 World Health Organization. Obesity and overweight [Internet]. Geneva: World Health Organization; 2018. Available from: https://www.who.int/news-room/fact-sheets/detail/obesity-and-overweight. 
Tran et al.: Global Evolution of Obesity Research in Children and Youths

11 World Health Organization. The global strategy on diet, physical activity and health (DPAS) [Internet]. Geneva: World Health Organization; 2004. Available from: https://www.who.int/nmh/wha/59/dpas/en/.

12 World Health Organization. Global action plan for the prevention and control of NCDs 2013-2020 [Internet]. Geneva: World Health Organization; 2014. Available from: https://www.who.int/nmh/events/ncd_action_ plan/en/.

13 Commission on Ending Childhood Obesity. Ending childhood obesity: securing the future for our children [Internet]. Geneva: World Health Organization; 2016. Available from: https://www.who.int/end-childhoodobesity/news/wha69-event/en/.

14 Müller O, Krawinkel M. Malnutrition and health in developing countries. CMAJ. 2005 Aug;173(3):279-86.

15 Schofield C, Ashworth A. Why have mortality rates for severe malnutrition remained so high? Bull World Health Organ. 1996;74(2):223-9.

16 Grimshaw JM, Eccles MP, Lavis JN, Hill SJ, Squires JE. Knowledge translation of research findings. Implement Sci. 2012 May; 7(1):50.

17 Bornmann L, Leydesdorff L. Scientometrics in a changing research landscape: bibliometrics has become an integral part of research quality evaluation and has been changing the practice of research. EMBO Rep. 2014 Dec;15(12):1228-32.

18 Sharifi V, Rahimi-Movaghar A, Mohammadi MR, Goodarzi RR, Izadian ES, Farhoudian A, et al. Analysis of mental health research in the Islamic Republic of Iran over 3 decades: a scientometric study. East Mediterr Health J. 2008 Sep-Oct;14(5):1060-9.

19 Eghbal MJ, Davari Ardakani N, Asgary S. A scientometric study of PubMed-indexed endodontic articles: a comparison between Iran and other regional countries. Iran Endod J. 2012;7(2):56-9.

20 Yazdani K, Nejat S, Rahimi-Movaghar A, Ghalichee L, Khalili M. Scientometrics: review of concepts, applications, and indicators. Iran J Epidemiol. 2015;10(4):78-88.

21 Gupta BM, Mueen Ahmed KK, Gupta R. A scientometric assessment of obesity research publications from India during 2007-16. OGH Rep. 2018;7(1):16-24.

22 Djalalinia S, Peykari N, Qorbani M, Moghaddam SS, Larijani B, Farzadfar F. Obesity researches over the past 24 years: a scientometrics study in Middle East countries. Int J Prev Med. 2015 May;6(1):38.

23 Aghaei Chadegani A, Salehi H, Yunus M, Farhadi H, Fooladi M, Farhadi M, et al. A comparison between two main academic literature collections: Web of Science and Scopus databases. Asian Soc Sci. 2013;9(5):18-26.

24 Yao Q, Chen K, Yao L, Lyu PH, Yang TA, Luo F, et al. Scientometric trends and knowledge maps of global health systems research. Health Res Policy Syst. 2014 Jun;12(1):26.

25 Wofford LG. Systematic review of childhood obesity prevention. J Pediatr Nurs. 2008 Feb;23(1):5-19.

26 Kesten JM, Griffiths PL, Cameron N. A systematic review to determine the effectiveness of interventions designed to prevent overweight and obesity in pre-adolescent girls. Obes Rev. 2011 Dec;12(12):997-1021.

27 Han JC, Lawlor DA, Kimm SY. Childhood obesity. Lancet. 2010 May;375(9727):1737-48.

28 Cornette R. The emotional impact of obesity on children. Worldviews Evid Based Nurs. 2008;5(3):136-41.

29 Tran BX, Pham TV, Ha GH, Ngo AT, Nguyen LH, Vu TT, et al. A bibliometric analysis of the global research trend in child maltreatment. Int J Environ Res Public Health. 2018 Jul;15(7):15.

30 Friedmacher F, Pakarinen MP, Rintala RJ. Congenital diaphragmatic hernia: a scientometric analysis of the global research activity and collaborative networks. Pediatr Surg Int. 2018 Sep;34(9):907-17.

31 Kearney J. Food consumption trends and drivers. Philos Trans R Soc Lond B Biol Sci. 2010 Sep;365(1554): 2793-807.

32 Ashton D. Food advertising and childhood obesity. J R Soc Med. 2004 Feb; 97(2):51-2.

33 Fryer CD, Ervin RB. Caloric intake from fast food among adults: United States, 2007-2010. NCHS Data Brief. 2013 Feb;(114):1-8.

34 Pirgon Ö, Aslan N. The role of urbanization in childhood obesity. J Clin Res Pediatr Endocrinol. 2015 Sep; 7(3): 163-7.

35 Matusitz J, McCormick J. Sedentarism: the effects of Internet use on human obesity in the United States. Soc Work Public Health. 2012;27(3):250-69.

36 Vuong QH. The (ir)rational consideration of the cost of science in transition economies. Nat Hum Behav. 2018; 2(1):5.

37 Quek YH, Tam WW, Zhang MW, Ho RC. Exploring the association between childhood and adolescent obesity and depression: a meta-analysis. Obes Rev. 2017 Jul;18(7):742-54.

38 Sjöberg RL, Nilsson KW, Leppert J. Obesity, shame, and depression in school-aged children: a population-based study. Pediatrics. 2005 Sep;116(3):e389-92.

39 Nemiary D, Shim R, Mattox G, Holden K. The relationship between obesity and depression among adolescents. Psychiatr Ann. 2012 Aug;42(8):305-8.

40 Mohammed Nawi A, Che Jamaludin FI. Effect of Internet-based intervention on obesity among adolescents in Kuala Lumpur: a school-based cluster randomised trial. Malays J Med Sci. 2015 Jul-Aug;22(4):47-56.

41 Gabriele JM, Stewart TM, Sample A, Davis AB, Allen R, Martin CK, et al. Development of an Internet-based obesity prevention program for children. J Diabetes Sci Technol. 2010 May;4(3):723-32.

42 Russell-Mayhew S, McVey G, Bardick A, Ireland A. Mental health, wellness, and childhood overweight/obesity. J Obes. 2012;2012:281801.

43 Griffiths LJ, Wolke D, Page AS, Horwood JP; ALSPAC Study Team. Obesity and bullying: different effects for boys and girls. Arch Dis Child. 2006 Feb;91(2):121-5. 